\title{
EFEITO DO PRÉ-TRATAMENTO ULTRASSÔNICO SOBRE A SECAGEM EM LEITO DE JORRO DE MISTURA CONSTITUÍDA POR RESÍDUO DE ACEROLA E SOJA
}

\author{
CUNHA $^{1}$, R.N.; OLIVEIRA ${ }^{2}$ O. S.; SILVA ${ }^{1}$ L.A.; DUARTE ${ }^{2}$, C.R.; BARROZO ${ }^{2}$, M. A. S. \\ ${ }^{1}$ Centro Universitário de Patos de Minas, Departamento de Engenharia Química \\ ${ }^{2}$ Universidade Federal de Uberlândia, Departamento de Engenharia Química \\ E-mail para contato: renatanepc@unipam.edu.br
}

\begin{abstract}
RESUMO - Os resíduos de acerola possuem elevados teores de ácido ascórbico e compostos bioativos. Testes preliminares de desidratação de resíduos de acerola em leito de jorro revelaram dificuldade na escoabilidade do material. Para garantir o bom comportamento fluidodinâmico e as características alimentícias do produto final, a soja foi selecionada como inerte. Elevadas temperaturas e tempos longos de operação podem afetar a qualidade nutricional do produto, entretanto, estudos apontam que a aplicação de ultrassom reduz o tempo de secagem. O presente trabalho teve como objetivo avaliar o efeito do pré-tratamento ultrassônico sobre a secagem e a qualidade do produto. A secagem e o comportamento fluidodinâmico foram caracterizados pela umidade e pelo índice de mistura. A qualidade do produto foi descrita pelos teores de: ácido ascórbico e cítrico, fenólicos e flavonoides. A partir da análise dos resultados foi possível avaliar o efeito do ultrassom sobre a secagem convencional.
\end{abstract}

\section{INTRODUÇÃO}

Pesquisas e avanços tecnológicos na esfera agroindustrial têm contribuído com o crescente aumento da produção de fruticultura brasileira. Em detrimento a esse fato resíduos provenientes desse setor são frequentes e merecem destaque pelo seu elevado volume.

O aproveitamento desses resíduos no processamento de novos alimentos tem representado um desafio importante para as indústrias, principalmente no tocante à demanda por produtos para dietas especiais (Santana, 2005). Santos et al. (2010) relata que os resíduos do processamento da acerola representam $40 \%$ do volume processado. Pesquisas envolvendo caracterização, secagem e reaproveitamento de resíduos de acerola se destacam no ramo alimentício devido ao seu significativo valor nutricional, rico em ácido ascórbico e compostos bioativos, dentre esses os fenólicos e os flavonoides (Borges, 2011; Jerônimo et al., 2002; Aguiar, 2010).

Estudos visando o uso do resíduo de acerola como ingrediente funcional, devido às suas propriedades antioxidantes foram realizados por diversos autores (Moreira, 2007, Borges, 2011; Duzzioni, 2010). Dentre as técnicas preservativas de alimentos com elevada umidade, a secagem é um 


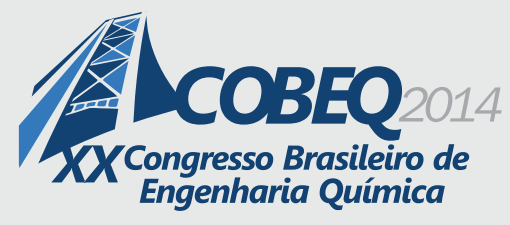

19 a 22 de outubro de 2014
Florianópolis/SC

dos processos comerciais mais indicados, fato esse aliado à simplicidade e ao baixo custo operacional, comparado a outros métodos preservativos como a centrifugação, os tratamentos químicos e a irradiação.

A desidratação de bagaços de acerola em leito de jorro foi conduzida por Borges (2011) que constatou a presença expressiva de compostos fenólicos totais e antocianinas, indicando que o resíduo pode ser considerado uma valiosa fonte de compostos bioativos. Estudos prévios de secagem de resíduo de acerola em leito de jorro revelaram a necessidade de adição de material auxiliar com o objetivo de contribuir na melhora da escoabilidade do material. Para garantir as características alimentícias do produto final, soja foi escolhida como inerte. Fatores benéficos como elevada esfericidade e sua dimensão contribuem na fluidodinâmica deste material em leito de jorro. A soja se caracteriza ainda por ser uma fonte rica em nutrientes, sendo passível a sua adição na formulação da farinha final (Bortolotti, 2012).

A aplicação de pré-tratamentos de secagem tem também tem sido objeto de estudo de vários autores (Fernandes e Rodrigues. 2007, 2012; Fernandes ett al, 2008; Rodriguez et al., 2014; Oliveira et al 2011; Pingret, 2013), dentre elas destacam-se: o ultrassom, a desidratação osmótica, e a desidratação mecânica. Neste âmbito, o ultrassom representa uma nova forma de exploração de energia limpa, que pode ser usada em diferentes segmentos, dentre os quais, o alimentício. Segundo a literatura o aumento de perda de umidade do fruto sujeito ao processo de pré-tratamento por ondas ultrassônicas se deve à ocorrência de uma série rápida de compressões e expansões que aumenta a transferência convectiva de massa no fruto, conduzindo a um processo de secagem mais rápido (Tarleton e Wakeman,1998; Fuente-Blanco et al, 2006).

Reduções significativas dos tempos de secagem foram obtidas na desidratação precedida por tratamento ultrassônico de mamão papaya (Fernandes et al, 2008), bananas (Fernandes e Rodrigues, 2007) e genipapos (Fernandes e Rodrigues, 2012), devido ao aumento da difusividade efetiva. Parâmetros como tempo e potência de sonicação podem exercer efeitos significativos sobre o processo ultrassônico e consequentemente sobre a secagem. Dentro desse contexto pretende-se por meio deste trabalho avaliar o efeito dessas variáveis na secagem e na qualidade nutricional do resíduo de acerola, quando submetido à secagem em leito de jorro.

\section{MATERIAIS E MÉTODOS}

Os resíduos de acerola foram provenientes da Nettare Indústria Comércio Importação e Exportação de Alimentos Ltda, empresa de processamento de frutas, e a soja utilizada no auxílio fluidodinâmico pertence à variedade Brazilian Doko.

As sonicações foram conduzidos em banhos ultrassônicos sem agitação mecânica. Para os ensaios a $80 \mathrm{~W}$, foram avaliados os tempos de sonicação de 10, 20, 30 50, 60 e 80 min, mantendo-se a proporção de água para resíduo de acerola de 4:1. Entretanto, para a condição de elevada potência (1050W) avaliou-se os tempos de 10, 30 e 60 min, utilizado-se água em excesso. Após a sonicação as amostras foram colocadas sobre papel absorvente para a retirada do excesso de água superficial. Um 


\section{9 a 22 de outubro de 2014 \\ Florianópolis/SC}

grupo controle foi estudado separadamente sem aplicação de ultrassom, para fins de comparação do efeito deste sob as demais amostras.

A secagem da mistura foi realizada em leito de jorro, adotando-se tempo de operação de 30min e altura de leito estático de $10 \mathrm{~cm}$. O jorro foi alimentado em regime batelada com composição de $45 \%$ de acerola, a qual foi inserida de forma aleatória e submetida à vazão de $20 \%$ acima da vazão de jorro mínimo e temperatura de $60^{\circ} \mathrm{C}$. Todos os ensaios foram realizados em duplicata.

Como variáveis definidoras da qualidade do produto final foram avaliadas: umidade, teores de ácido cítrico e ascórbico e compostos bioativos fenólicos e flavonoides, sendo as análises realizadas em triplicata. As avaliações microestruturais foram realizadas por meio de fotomicrografias em microscópio eletrônico de varredura (MEV).

\subsection{Análises de compostos bioativos}

2.1.1 Análise de acidez total titulável: realizada por titulometria utilizando solução de hidróxido de sódio a 0,1N, segundo o método da Association of Official Analytical Chemists (1995). Os resultados encontram-se expressos em $\mathrm{mg}$ de ácido cítrico/100 $\mathrm{g}$ amostra em base seca.

2.1.2 Análises de ácido ascórbico: o teor de vitamina C expresso em $\mathrm{mg}$ de ácido ascórbico/100mg de amostra seca foi mensurado por titulação baseada na redução de 2,6-diclorofenol-indofenol pelo ácido ascórbico (AOAC, 1995).

2.1.3 Determinação dos teores de fenólicos totais: quantificação realizada pelo método de FolinCiocalteu usando ácido gálico como padrão e leitura da absorbância a $622 \mathrm{~nm}$. Os resultados obtidos são expressos em mg de ácido gálico em $100 \mathrm{~g}$ de amostra seca.

2.1.4 Determinação do teor de flavonoides totais: extração efetuada com metanol de acordo com Yu e Dahegren (2000) e determinado pelo método colorimétrico segundo Zhishen et al. (1999), com leitura de absorbância a $450 \mathrm{~nm}$. Os resultados são expressos em mg de rutina/100 g de amostra seca.

\section{RESULTADOS E DISCUSSÕES}

Objetivando-se avaliar o efeito do pré-tratamento ultrassônico sobre a secagem e a qualidade nutricional do resíduo de acerola, os ensaios de sonicação foram conduzidos com variações de tempo e de potência. Para fins de comparação adotou-se como grupo controle a secagem convencional, ou seja, sem aplicação de ultrassom.

Dentro desse contexto, a eficiência da secagem avaliada pela redução de umidade, calculada em relação à umidade inicial do resíduo é apresentada na Figura 1. Analisando-se a Figura 1 verifica-se que a secagem convencional reduziu o teor de umidade em $85,2 \%$. Os resultados sugerem que o uso de ultrassom influencia a secagem do produto, pois para todas as condições avaliadas obtiveram-se reduções de umidade superiores à do controle.

Verifica-se que reduções significativas de umidade $(89,2 \%)$ foram obtidas após sonicação de 50 min à $80 \mathrm{~W}$ (Figura 1). Atribuem-se esses resultados à alteração da camada limite entre o resíduo de acerola e o meio líquido e à possível formação de canais microscópicos, causados pela aplicação de 
ultrassom, decorrentes dos fenômenos de cavitação e efeito esponja. Tais resultados estão em concordância com Fernandes e Rodrigues $(2007,2012)$ o qual afirma que o tempo de ultrassom afeta o coeficiente difusivo de transferência de massa e consequentemente o tempo de secagem ou a redução de umidade.

Para a maioria dos tratamentos nota-se que a elevação da potência não promoveu alterações significativas na secagem. Tal fato se confirma pela análise de variância (ANOVA) na qual se verifica que o tempo afeta significativamente a secagem, no entanto a potência não exerce efeito significativo sobre essa resposta. Estudos de secagem precedida por ultrassom realizados por Sarabia et al. e Juárez et al. (2006) indicaram que o aumento da potência ultrassônica favoreceu a taxa de secagem de cenouras e cascas de limão. A diferença entre estes resultados pode ser devido às características estruturais dos materiais.

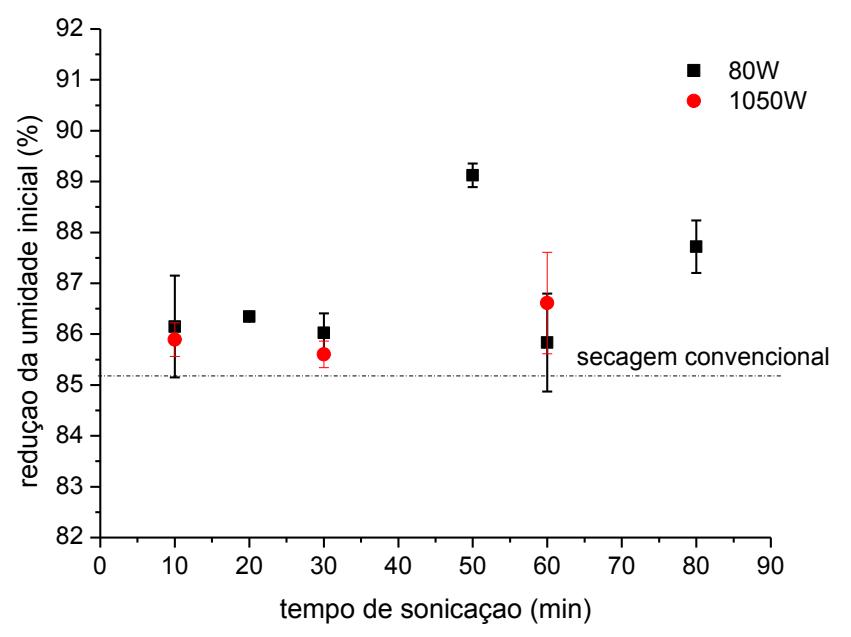

Figura 1 - Avaliação do pré-tratamento ultrassônico sobre a redução de umidade durante a secagem.

As alterações causadas pelo ultrassom na qualidade nutricional do resíduo em relação à secagem convencional são apresentadas nas Figuras 2 e 3. Verifica-se pela Figura 2A que a sonicação acarretou, para a maioria dos tratamentos, reduções na acidez do produto. Considerando que o ultrassom degrada a matéria orgânica, levando à produção de ácidos, como fórmico e acético (Yousef, 1999) seria de se esperar um aumento de acidez titulável, uma vez que o valor desta se relaciona à concentração total de ácidos na amostra (Rodriguez, 2014). No entanto, cabe ressaltar que o ácido cítrico possui elevada solubilidade em água, o que justifica seu decréscimo. De modo similar, a solubilidade do ácido ascórbico em água explica as reduções obtidas em todos os tratamentos com ultrassom, quando comparados com a secagem sem pré-tratamento (Figura 2B).

Nota-se que para a maioria dos tratamentos (Figuras 2A e 2B) as maiores reduções foram obtidas durante a aplicação de potência mais elevada, o que sugere a influência desta variável sobre as respostas. As análises de variância revelam que as duas variáveis exercem efeito significativo $(\mathrm{p}<0,05)$ sobre a acidez, no entanto, neste nível de significância, apenas o tempo exerce efeito significativo sobre o teor de ácido ascórbico. 
Constatam-se pela (Figura 3A) reduções nos teores de fenólicos totais para todos os tratamentos, o que possivelmente se deve à degradação desses compostos. Resultados similares foram obtidos em secagem de maçãs, onde perdas significativas de fenólicos e flavonoides foram obtidas em condições de elevada intensidade de ultrassom (Rodrigues, 2014).
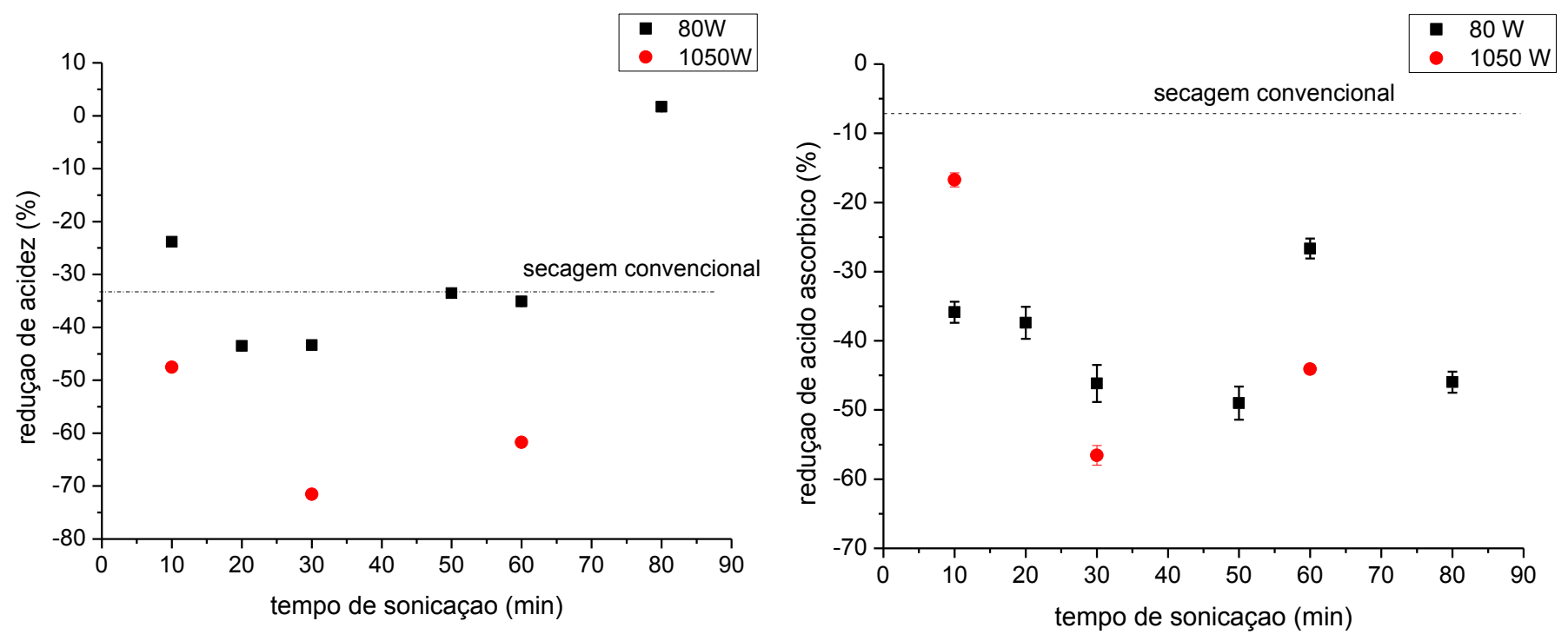

Figura 02 - Teores de acidez (A) e ácido ascórbico (B) em relação à secagem convencional
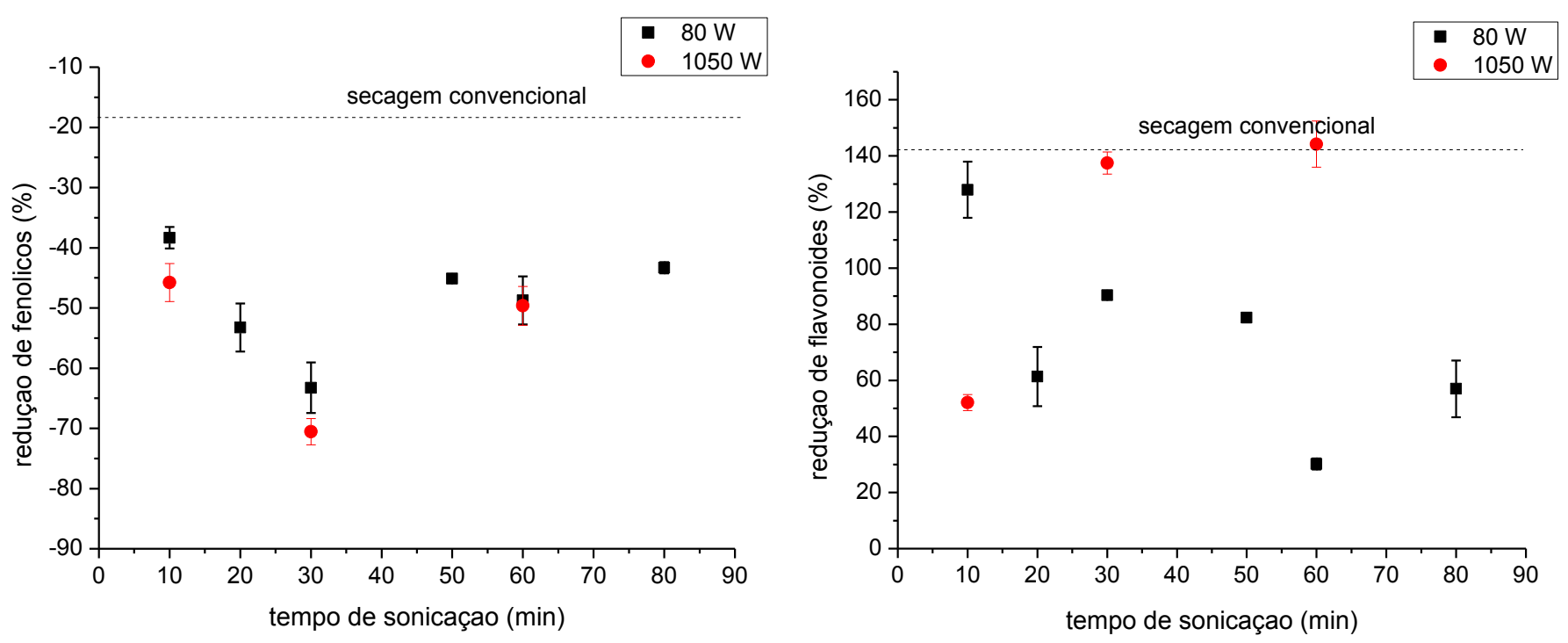

Figura 03 - Teores de (A) fenólicos e flavonoides (B) em relação à secagem convencional.

Avaliações estatísticas apontam que o tempo exerce efeito sobre os fenólicos, o que não ocorre para os teores de flavonoides, onde nenhuma das variáveis em questão exerce efeito significativo 
sobre essa resposta, para um nível de significância de $5 \%$.

Para se observar as possíveis alterações estruturais causadas pelo tratamento ultrassônico no tecido estrutural do resíduo de acerola, utilizou-se a microscopia eletrônica de varredura. Desse modo avaliaram-se as imagens de amostras resultantes da secagem convencional e da secagem precedida por tratamento de ultrassônico.

Conforme mostra a micrografia eletrônica de varredura da amostra resultante da secagem convencional (Figura 4A) o tecido do resíduo apresentou células túrgidas com parede celular definida e organizada. Quando comparado à secagem convencional verifica-se que a sonicação promoveu alterações na estrutura do resíduo, pois observam-se distorções das paredes celulares e possíveis formações de microcanais (Figura 4B e 4C).
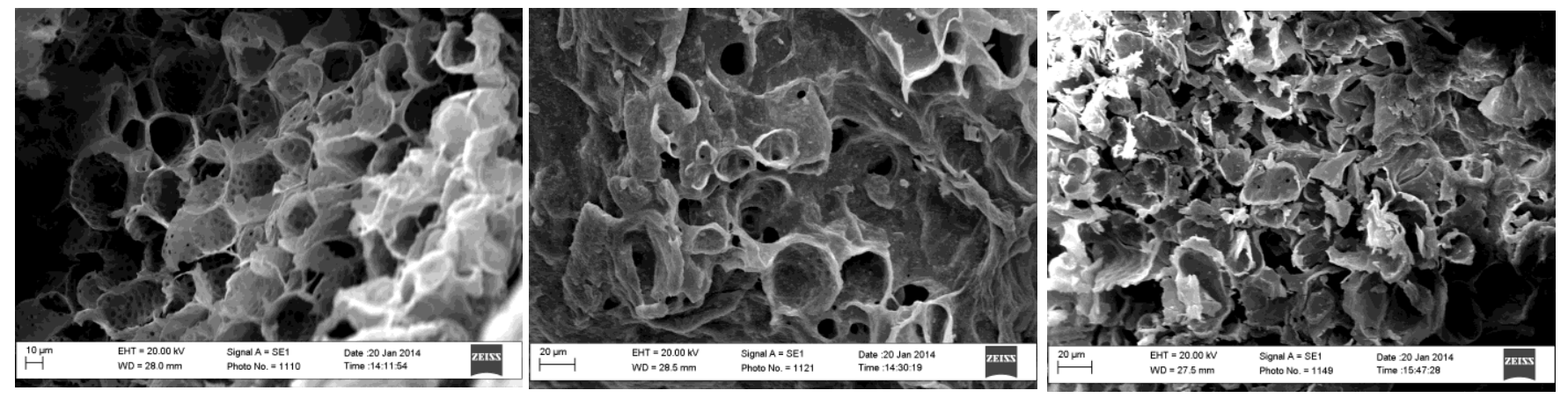

Figura 4 - Fotomicrografias provenientes da secagem. (A) convencional, (B) 30min de ultrassom e (C) 50min de ultrassom.

\section{CONCLUSÕES}

De acordo com os resultados obtidos, pode-se concluir que, de um modo geral, o uso do ultrassom favoreceu a secagem, quando se compara a redução de umidade do resíduo de acerola, proveniente do pré-tratamento ultrassônico com a secagem convencional, nas mesmas condições operacionais.

Reduções significativas foram obtidas para os teores nutricionais, dentre eles a acidez, o teor de ácido ascórbico e de compostos bioativos: fenólicos e flavonoides. Dentre as variáveis estudadas o tempo de sonicação exerceu efeito significativo sobre a maioria dos parâmetros tidos como objeto de estudo.

Alterações estruturais nas paredes celulares dos resíduos de acerola foram obtidas quando os mesmos se submeteram ao processo de ultrassom, fato esse que, provavelmente, pode ter contribuído para a alteração nas variáveis estudadas neste trabalho.

\section{AGRADECIMENTOS}




\section{9 a 22 de outubro de 2014 \\ Florianópolis/SC}

Agradecemos ao CNPQ e a Fundação de Amparo à Pesquisa do Estado de Minas Gerais (FAPEMIG) pelo apoio Financeiro para a realização da pesquisa e ainda a FAPEMIG pelos recursos concedidos no Projeto de Participação Coletiva em Eventos Técnicos-Científicos (PCE00082-14). Em especial ao Laboratório de Microscopia pela colaboração no desenvolvimento deste trabalho.

\section{REFERÊNCIAS}

AGUIAR, T. M.; RODRIGUES, F. S.; SANTOS, E. R.; SABAA-SRUR, A. U. O. Caracterização química e avaliação do valor nutritivo de sementes de acerola. Nutrire., v. 35, p. 91-102, 2010.

ASSOCIATION OF OFFICIAL ANALYTICAL CHEMISTS-AOAC. Official methods of analysis. Washington: DC, 1995.

BORGES, K.C. Estudo das características físico-químicas e funcionalidade de bagaços de frutas tropicais desidratados em leito de jorro. Dissertação de mestrado. Pós-graduação em Engenharia Química do Rio Grande do Norte, 2011.

BORTOLOTTI, C.T. Estudo experimental da fluidodinâmica de uma mistura de resíduo de acerola e soja em leito de jorro. Dissertação (mestrado) Universidade Federal de Uberlândia, Programa de Pós-Graduação em Engenharia Química. 101p, 2012.

DUZZIONI, A. G., FRANCO, A. G., DUZZIONI, M., SYLOS, C. M. Determinação da atividade e de constituintes bioativos em frutas cítricas. Alim. Nutr. v. 21, p. 643-649, 2010.

FERNANDES, F. A.N.; RODRIGUES, S. Ultrasound as Pre-Treatment for Drying of Genipap (Genipa americana L.). Inter. Journal of Food Engineering, v.8, 2012.

FERNANDES, F. A.N.; RODRIGUES, S. Ultrasound as pre-treatment for drying of fruits: Dehydration of banana. Journal of Food Engineering, v. 82, p. 261-267, 2007.

FERNANDES, F.A.N.; GALLA, M.I.; RODRIGUES, S. Effect of osmotic dehydration and ultrasound pre-treatment on cell structure: Melon dehydration. Society of Food Science and Technology, p. 604-610, 2008.

FUENTE-BLANCO, S.; SARABIA, E. R. F.; ACOSTA-APARICIO, V. M., LANCOBLANCO, A.; GALLEGO-JUAREZ, J. A. Food drying process by power ultrasound. Ultrasonics Sonochemistry, v. 44, p. 523-527, 2006.

GONZE, E. et al. Wastewater pretreatment with ultrasonic irradiation to reduce toxicity. Chemical Engineering Journal, v.73, p. 93-100, 1999.

JERÔNIMO, C.E.M; CEZAR, G.M.;SANTIAGO JÚNIOR, A.F.; OLIVEIRA, V.G.; MELO, H.N.S. Caracterização dos resíduos das indústrias potiguares de beneficiamento de polpas de frutas. VI Simpósio Ítalo Brasileiro de Engenharia Sanitária e Ambiental , 2002. 
JÚAREZ-GALlEGO, J.A., BLANCO-BLANCO, A., ACOSTA-APARICIO, V.M., RIERAFRANCO DE SARABIA, E., DE LA FUENTE-BLANCO, S. Food drying process by Power ultrasound. Ultrasonics. v. 44, p. 523-527, 2006.

MOREIRA, E.G. Obtenção e caracterização de extrato microencapsulado de resíduo agroindustricial de acerola. Dissertação de Mestrado. Universidade Federal do Rio Grande do Norte, 86p, 2007.

OLIVEIRA, F.I. P. et al. Dehydration of Malay Apple (Syzygium malaccense L.) Using Ultrasound as Pre-treatment. Food Bioprocess Technol, v.4, p. 610-615, 2011.

PINGRET D.; SYLVIE A. TIXIE F.; CHEMAT F. QIAO L.; YE X.; SUN Y.; YING J.; SHEN Y.; CHEN J. Sonochemical effects on free phenolic acids under ultrasound treatment in a model system. Ultrasonics Sonochemistry, v. 20, p.1017-1025, 2013.

RODRIGUEZ O.; SANTACATALINA J.V.; SIMAL S.,GARCIA-PEREZ J.V.; FEMENIA A, ROSSELO C. Influence of power ultrasound application on drying kinetics of apple and its antioxidant and microstructural properties. Journal of Food Engineering, v. 129,p. 21-29, 2014.

SANTANA, M. F.S. Caracterização físico-química de fibra alimentar de laranja e maracujá. Tese de Doutorado em Engenharia de alimentos. Faculdade de Engenharia de alimentos, Universidade Estadual de Campinas.168p, 2005.

SANTOS, K. O.; NETO, B. A. M.; OlIVEIRA, S.; RAMOS, M. E. C.; AZEVEDO, L. C. Obtenção de Farinha com o Resíduo da Acerola (Maplpighia Glabra L.). Congresso de Pesquisa e Inovação da rede Norte Nordeste de Educação Tecnológica, 2010.

SARABIA, R.F., DE LA FUENTE-BLANCO, S., CÁRCEL, J.A., GARCÍA-PÉREZ, J.V. Ultrasonic drying of foodstuff in a fluidized bed: Parametric study. Ultrasonics. V. 44, p. 539$543,2006$.

TARLETON, E. S.; WAKEMAN, R. J. Ultrasonically assisted separation process. Ultrasounds in food processing. p. 193-218, 1998.

YOUSEF, A. E; KIM, J. G.; DAVE, S. Application of ozone for enhancing the microbiological safety and quality of foods: a review. Journal of Food Protection, v.62, p. 1071-1087, 1999.

YU, D.; DAHEGREN, R.A. Evaluation of methods for measuring polyphenol in coniter foliage. J. Chem. Ecology, v.26, p. 2119-2140, 2000

ZHISHEN, J.; MENGCHENG, T.; JIANMING, W. The determination of flavonoid contents in mulberry and their scavenging effects on superoxide radicals, Food Chemistry, v. 64, p.555-559, 1999. 